\title{
Modelling and Evaluation of a Policy-Based Resource Management Framework for Converged Next Generation Networks
}

\author{
S. Y. Yerima, G.P. Parr, S. McCLean, P. J. Morrow \\ School of Computing and Information Engineering, \\ University of Ulster, Northern Ireland \\ Email: \{s.yerima, gp.parr, si.mcclean,pj.morrow\}@ulster.ac.uk
}

\begin{abstract}
As fixed and wireless access networks converge towards Internet Protocol based transport in next generation networks, the requirement for effective and scalable control and management solutions to address the complexity introduced by their heterogeneity becomes ever more critical. Over the years, policy-based management has emerged as a viable tool to address this challenge. Within the IU-ATC project, we are developing a policy-based framework, (Converged Networks QoS Framework, CNQF) for end-to-end QoS control and resource management in converged next generation networks. CNQF is designed to support QoS control and context-driven resource management policies across all networks (access, metro, core) on the end-to-end transport layer of converged networks. This paper describes CNQF and develops an exemplary use case scenario for policybased resource management based on our CNQF architecture. The paper also presents the development of stochastic-analytic models to characterise and evaluate the impact of policies on operational performance. The model is used to analyse the performance of dynamic CNQF context-driven policies for admission control case study.
\end{abstract}

Index Terms-QoS, Context, Policy-based management, converged networks, resource management, admission control

\section{INTRODUCTION}

The need to offer guaranteed end-to-end Quality of Service (QoS) to end-users while effectively managing network resources is becoming more crucial as operators move towards transforming existing infrastructures to converged fixedwireless IP-based networks. In converged networks, a common core network $(\mathrm{CN})$ typically provides the means to interconnect different wired and wireless access networks based on the Internet Protocol (IP). Thus, IP technology is becoming the cornerstone around which various access technologies are converging [1]. Traditional IP networks where service provision was "best-effort" were inadequate to meet the QoS requirements imposed by real-time applications such as VoIP, streaming video, IPTV, and other real-time multimedia applications. This prompted the standardisation of IP QoS technologies such as IETF DiffServe and IntServe to provide service guarantees in IP-based networks.

Even where IP QoS mechanisms are deployed in converged networks, there is also a requirement to support and integrate often incompatible QoS mechanisms in different wireless and wireline access technologies in order to achieve end-to-end QoS provisioning. Also, the steady evolution of these access technologies to meet growing demand in services and to support various forms of mobility (service, terminal, session) demands effective solutions for their integrated control and management within converged networks architectures. A homogeneous framework is needed in which applications and content providers can obtain appropriate resources for their services in accordance with Service Level Agreements (SLA). Policybased management is emerging as an important and effective tool to address these challenges. This is evident from ongoing efforts (for example [2], [3], [4]) by standards bodies such as IETF, ETSI TISPAN, 3GPP etc., aimed at specification and refinement of policy-based control and management architectures to meet the challenges imposed by convergence in next generation networks. Policy-based management is the key to application transparency across existing and emerging access technologies, thus permitting applications to be agnostic to the transport layer in next generation networks.

Within our project, we leverage a policy-based management paradigm to develop a context-aware policy-based framework for QoS control and resource management in converged next generation networks. The policy-based framework, Converged Networks QoS Framework (CNQF), is designed to provide homogeneous, unified, end-to-end QoS support over heterogeneous access technologies, together with scalable, contextaware resource control and adaptive QoS control [5]. CNQF will benefit operators through providing critical policy control and adaptation functionality needed to deliver a wide variety of high-value services with guaranteed QoS across fixed and wireless access technologies. Furthermore the incorporation of context-aware resource control functionality within CNQF will provide value-added capability to intelligently manage network resources dynamically and in real-time thereby increasing revenue for network providers.

This paper presents a use case scenario for CNQF resource 
management in converged networks. The case study considers policy-based admission control in an exemplary CNQF 'managed resource', e.g. an MPLS pipe provisioned by an operator to handle various classes of traffic for different heterogeneous access domains which may be owned and operated by different administrative entities from the provider of the (core network) MPLS pipe. This paper also contributes to the literature on analytical modelling and evaluation of policy based management entities through development of stochastic analytic models to characterise and evaluate the CNQF policies considered in the case study scenario. Such models provide viable but cost-effective performance evaluation to complement simulation and/or experiments on live testbeds.

The rest of the paper is organized as follows. Section II discusses background and motivation for our work. Section III summarizes the CNQF architecture and functionality. Section IV presents the CNQF use case scenario, model formulation and evaluation. Section $\mathrm{V}$ concludes the paper and highlights ongoing and future work within our project.

\section{BACKGROUND AND MOTIVATION}

Policy-based management is a means to equip networks with the ability to provide an automatic response to network conditions according to pre-defined policies, and is considered the best approach to ease the complex network configuration process involved in the integration of network services in to a single large network [6], [7]. Various architectures have been developed for policy control within the standards forums; for example, Telecoms and Internet Converged Services and Protocols for Advanced Networks (TISPAN) technical committee of the European Telecommunications Standards Institute (ETSI), Third Generation Partnership Project (3GPP) and the Internet Engineering Task Force (IETF). The standards architectures define function blocks, interfaces and protocols which allow for interoperability between products from different vendors. TISPAN and 3GPP policy-based architectures share some similarities in the employment of policy decision entities which act as policy servers for the policy enforcement points in the transport plane. For example, TISPAN defines a Servicebased Policy Decision Function (SPDF) as part of its Resource and Admission Control Subsystem (RACS) while 3GPP Release 5/6 policy framework defines a Policy Decision Function (PDF). Both entities serve as policy servers to policy enforcement points on the managed network elements. Indeed, these architectures align with the original IETF policy based model which specified: a Policy Decision Point (PDP), Policy Enforcement Point (PEP), policy repository, and policy management tool. The design of our CNQF is also based on a similar architectural model.

Despite defining function blocks, interfaces, and protocols, the standards do not include details of how the functions will be implemented or how for example information is obtained and stored. Thus, the standards in themselves do not provide complete comprehensive solutions; hence, our motivation for developing CNQF which leverages aspects of the standards' architectural model while providing important innovative extensions to enable context-driven end-to-end resource control in NGNs.

Aligning CNQF design with the standards' architectural model is a deliberate design choice to facilitate its extensibility and interoperability with other standards based protocols, mechanisms and subsystems which may provide services outside the scope of CNQF QoS architecture (for example IMS-based services). The CNQF architecture defines how policy interacts with the transport network to enable dynamic policy-based resource control as a tool for delivering the QoS required by applications. Specifically, the architecture comprises three key subsystems, which have interfaces to the transport network(s). These are: Resource Management Subsystem (RMS); Context Management and Adaptation Subsystem (CAS); and Measurement and Monitoring Subsystem (MMS). Essentially, the incorporation of CAS and MMS into CNQF framework design provides a closed-loop, policy-based QoS control and contextdriven adaptation framework to enable more intelligent and adaptive service support beyond the capabilities that are possible with current standards architectures.

There are several related works to our project on policybased QoS and resource management in the literature. Kim et al., for example, present a QoS framework in [8] based on DiffServe and Simple Network Management Protocol (SNMP) designed to provide QoS control in ad hoc military environments. Similarly, in [9] a policy-based multi-layer QoS architecture for network resource control based on Traffic Engineering (TE) and policy-based routing is presented. Other works addressing policy-based resource management can be found in [10], [11], [12] and [13], to mention a few. These works however, like majority that can be found in the literature, lack support for context-driven policies or context-aware mechanisms in their solutions; unlike our proposed CNQF where such support is considered an important enabling feature to provide more intelligent and adaptive QoS control.

Although some papers such as [14], [15], and [16] consider context-aware service provisioning, unlike our proposed solution, their studies did not address end-to-end QoS provisioning in converged networks. Another way that the work in this paper differs from the aforementioned is that it explores the modelling of policy-driven processes using stochastic-analytic tools (specifically Markov Chains) as a cost-effective method to evaluate the impact of context-driven policies in a CNQF case study scenario (in section IV).

\section{CNQF ARCHITECTURE}

The Converged Networks' QoS Framework (CNQF) designed within our project is made up of three logical subsystems: Resource Management Subsystem (RMS); Context Management and Adaptation Subsystem (CAS); and Measurement and Monitoring Subsystem (MMS). Together, these three subsystems provide the policy-based infrastructure to 
enable closed-loop, scalable, end-to-end QoS control and resource management in converged next generation networks.

\section{A. Resource Management Subsystem (RMS)}

The primary responsibility of the RMS is providing joint allocation, co-ordination and control of the resources in the transport layer in accordance with customer SLAs. In the design of RMS, we take into account the requirement of CNQF to be deployable not only in single converged network environments (i.e. those where access and core networks are owned or operated by the same provider), but also multi-network deployment where each network (access, metro, core) could be owned and operated by different business entities. Thus, RMS is made up of distributed instances of peer resource brokers (RB) which act as Policy Decision Points (PDPs) within each network present on the end-to-end transport plane (see Fig. 1).

Thus, the role of a CNQF RB will vary depending on the type of network that it is configured to act as PDP for. For example, in a wireless access network such as WiMAX, the RB present is designated Wireless Access Resource Broker (WARB) and will be responsible for policies that co-ordinate the resources in the wireless access portion of the converged network. Such policies will include local and global admission control and bandwidth management policies. Likewise, for wired access networks such as Optical networks or xDSL, Fixed Access Resource Brokers (FARB) will be the designated PDP to perform a similar role. For the core network(s), an RMS RB will be designated Core Network Resource broker (CNRB). The CNRB will interface with the various WARBs and FARBs that make policy decisions for the access networks connected to the core network being overseen by the CNRB. This is important because CNRB will be responsible for re- source brokerage between the access RBs which allows for scalable management of resources on an end-to-end basis.

Another key entity of the RMS is the set of Resource controllers (RCs) which are the logical management and control entities responsible for low level (re)configuration at the Policy Enforcement Points (PEPs) in the transport plane. The PEPs are at the network entities such as gateway nodes, access routers, edge routers etc. where the policy decisions made in the PDPs (i.e. RBs) are enforced. Thus each RB (WARB, FARB, CNRB) is interfaced with one or more corresponding RCs (FARC, WARC, CNRC) which perform different functions depending on where the PEPs are located on the transport plane. For instance, in the core network an RC located in the edge router (PEP) may be responsible for packet marking (for example DSCP marking in a DiffServe domain) in response to policy decisions made in the CNRB. While in the wireless access network, an RC may be responsible for configuration of RAN elements such as a Gateway GPRS Support Node (GGSN) for UMTS/HSPA access network.

\section{B. Monitoring and Measurement Subsystem (MMS)}

The ability to monitor the conformance with CNQF policy decisions in the underlying converged networks being managed is vitally important to the efficient operation of the CNQF framework. Likewise, passive and active measurements at strategic points within the converged transport plane at required times are essential to policy-based QoS control with CNQF. These capabilities are enabled by the MMS which allows CNQF to gauge performance and adapt configurations to enable dynamic resource management. The MMS consists of network monitoring entities (NMs) which are located at the PEPs for monitoring and measurement collection.

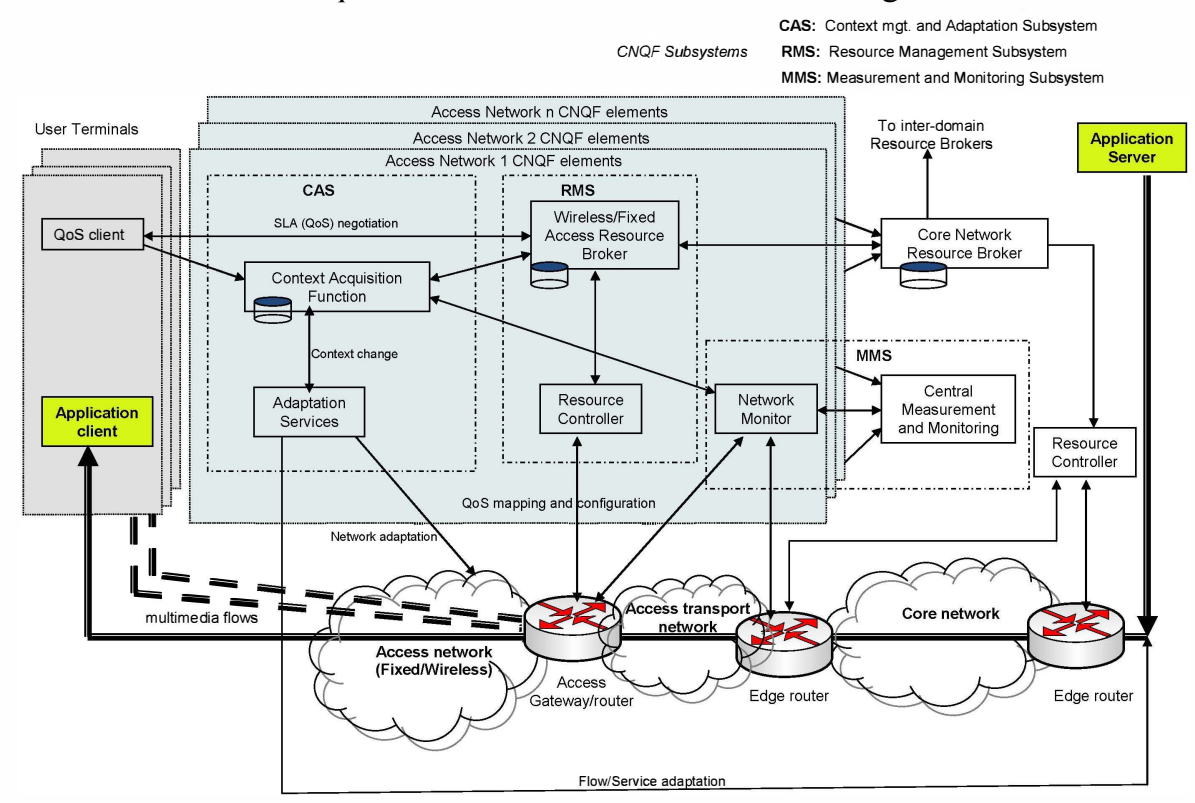

Fig. 1. CNQF operational entities in a converged network [5]. 


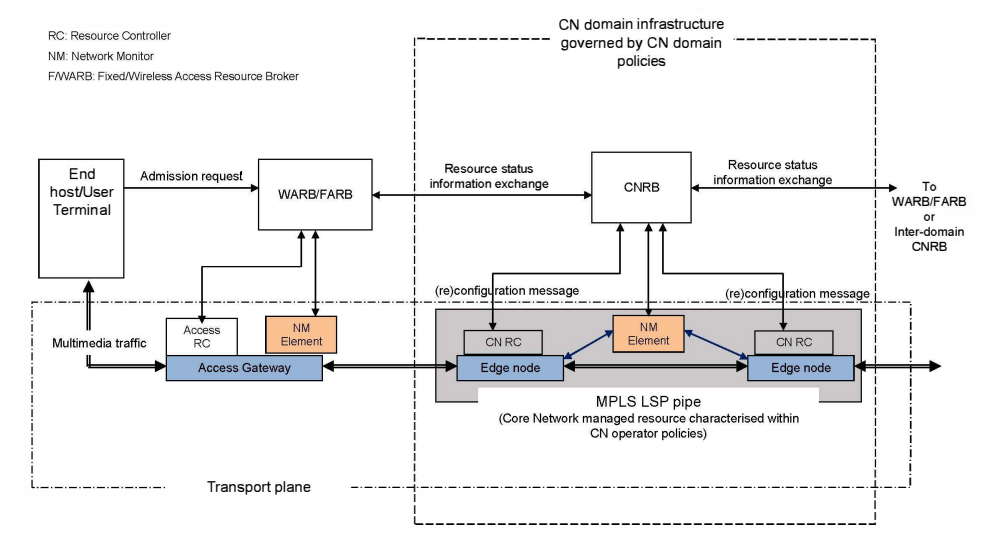

Fig. 2. CNQF use case scenario: MPLS LSP resource management for policy-based admission control.

The current implementation of our CNQF consists of NMs that use SNMP for monitoring at PEPs using SNMP MIBs. NMs interface with a central measurement and monitoring server (MMS) which serves as aggregating entity for the entire MMS. The measurements provided by NMs may be utilized by the RMS and CAS subsystems via interfaces to the MMS.

\section{Context Management and Adaptation Subsystem (CAS)}

Nowadays, the concept of 'context-awareness' is growing in importance within networking research. In [17], context is defined as 'any information obtained either implicitly or explicitly that can be used to characterise one certain aspect of an entity involved in a specific application or network service.' Policy-based network management infrastructures, such as $\mathrm{CNQF}$, stand to benefit from the use of context information to drive policies/policy adaptation. This equips the management system with increased intelligence and ability to adapt service provision, resource allocation, and QoS control in a more flexible and efficient manner. Furthermore, context-awareness introduces more flexibility and autonomy to the CNQF system so as to respond to highly dynamic operational conditions.

In order to facilitate 'context-aware' functionality in the $\mathrm{CNQF}$, appropriate infrastructure to gather, process, manage and disseminate context information is needed. Thus, CNQF meets this requirement through its Context Management and Adaptation Subsystem (CAS). CAS consists of distributed Context Acquisition Function blocks (CAF) which will be present in each access network. The CAFs are PDPs that execute context-aware or context-driven policies within the CNQF system. Each CAF elements has associated Adaptation Servers (ADs) which are function blocks that configure/reconfigure PEPs that are directly affected by contextdriven policy decisions in the CAF. CAFs also interface to the RBs in the RMS subsystem to facilitate context-driven resource control. CAFs may also interface to the MMS to request measurements that allows it to infer (network-centric) context information from such measurements.

Entities characterised by context could be physical objects e.g. a user device, router, physical link, wireless channel; or could be a virtual object such as MPLS path, VPN tunnel, IP-
Sec tunnel, an agent or even a protocol whose parameters may be configured in response to different 'contexts'. Thus, depending on the policies in operation, a CAF may be required to process information from several context sources. Contextdriven configuration changes may be undertaken by the appropriate ADs in the PEP where a specific CAF policy applies. For example, reconfiguring appropriate wireless access network (RAN) nodes to execute (network-centric) vertical handover management policies which may be driven by context information such as user profile, location, speed, etc; or reconfiguring a protocol parameter according to a user or device context.

\section{CASE STUDY: CONTEXT-AWARE POLICY BASED ADMISSION CONTROL WITH CNQF}

In this section, a use case scenario for CNQF policy-based resource management in converged networks is presented. In particular, we exemplify, model and evaluate CNQF application for context-driven policy-based admission control within a 'managed resource' of the core network domain; in this case, a hypothetical MPLS pipe (Label Switched Path, LSP) as shown in Fig. 2. We develop stochastic-analytic models to characterise the behavior of the Policy Decision Points (PDP) within CNQF. The models are based on Continuous Time Markov Chains (CTMC) which provides a useful, cost-effective tool for analysis of the impact of policies on operational performance as they are able to capture state transitions within the PDPs that may be triggered by events external to the PDP or the outcome(s) of evaluation of policy conditions.

\section{A. Admission control process}

Admission control is a vital mechanism that allows for SLA to be guaranteed and maintained for end users. Without admission control process, the QoS of the users in a session might be compromised if the network admits more sessions than the end-to-end resource capabilities can handle. The admission control process in the CNQF RMS policies may be made more dynamic if 'context information' is used to characterise the managed resources within the high-level admission policy rules leading to more efficient network resource utilisation.

As highlighted in section III, CNQF operation in a con- 
verged network enables end-to-end admission control through co-ordination between the various RBs in the RMS. For example, a WARB/FARB responsible for admission control in the wireless/wired access network in response to an end user request may solicit resource availability information in the transiting $\mathrm{CN}$ domain from the CNRB which in turn can request the state of resource availability in the destination access network or other transiting CNs (see Fig. 2). Note that the $\mathrm{CN}$ resources may belong to a different administrative domain, and as such its corresponding CNRB would not process admission requests from end users but may instead receive requests for resources from attached WARBs, FARBs or CNRBs associated with other (transit) CNs. Hence, CNRB does not need to manage per flow QoS but will provide aggregated QoS management while WARBs and FARBs could be configured to provide per flow QoS control. This design enables CNQF RMS to provide scalable end-to-end policy-based resource control. A high-level policy rule for global admission control may be expressed as follows (within WARB/FARB):

\section{If $\left\{\right.$ min $\left.\left(B W_{\text {access, }}, B W_{\text {core }}, \ldots\right)>B W_{\text {req }}\right\}$ then admit end user request else reject end user request}

Where $\min \left(B W_{\text {access }}, B W_{\text {core }}, \ldots\right)$ indicates the end-to-end availability of bandwidth along the various transiting networks (access, core) to service the end user request $\left(B W_{\text {req }}\right) . B W_{\text {core }}$ indicates the available bandwidth in the $\mathrm{CN}$ domain which is governed by a different set of policies than those used to determine $B W_{\text {access }}$ (available bandwidth in the access network domain) especially where both constitute separate administrative domains.

\section{B. Context-driven policies in the core network}

Suppose the MPLS LSP shown in Fig. 2 is provisioned in the $\mathrm{CN}$ by the operator to handle traffic of various pre-defined classes $\mathrm{C}_{1}, \mathrm{C}_{2} \ldots \mathrm{C}_{\mathrm{N}}$. Based on the definition of context given earlier in section III C, context information may be used to characterise this managed resource (i.e. LSP) in the CNRB policies to allow for adaptation of the resource allocation process. The context-aware $\mathrm{CN}$ policies involved in the end-toend admission control would be modeled with the policy objective: to maximise edge-to-edge (LSP) bandwidth utilisation whilst ensuring minimum bandwidth reservation for each class of traffic. Under assumption of DiffServe-aware MPLS, we define 3 service classes $C_{1}$ (voice), $C_{2}$ (video) and $C_{3}$ (data) for the LSP.

Context is usually defined by a set of variables that characterise the state of the managed object allowing for different sets of conditions within the policies to be applied for each 'context' (i.e. context-aware policies). For the scenario under consideration, the variables employed are voice utilisation, video utilisation and data utilisation which can assume either state 'high' or 'low' to characterise the LSP bandwidth consumption per class of traffic within the high-level policies. The 'utilisation context' will therefore determine which set of actions are applied by the policy rules.
Suppose that operator policy rules apply a default admission threshold when utilisation context is high while an optimised admission threshold is applied when utilisation context is low. Thus, a set of CNRB high-level declarative policies that enable context-driven allocation could be expressed as follows:
Rule 1: If (video utilisation $==$ low and data utilisation $==$ low) set (voice admission threshold $=$ 'optimised') else set (voice admission threshold $=$ 'default')
Rule 2: If (voice utilisation $==$ low and data utilisation $==$ low) set (video admission threshold = 'optimised') else set (video admission threshold = 'default')
Rule 3: If (voice utilisation $==$ low and video utilisation $==$ low) set (data admission threshold $=$ 'optimised') else set (data admission threshold $=$ 'default')

This set of policies corresponds to the following expression:

For each class $i$, where $i \neq k$ :

IF $\sum_{k=1}^{N} \mathrm{~B}_{k}(\mathrm{t})=$ low THEN $\mathrm{B}_{i}=\mathrm{B}_{\mathrm{T}}-\sum_{i=1}^{N} \mathrm{~B}_{i}(\mathrm{t})$ ELSE $\mathrm{B}_{i}=\mathrm{B}_{i R E S}$

Where $N$ is the number of pre-defined network service classes; $\mathrm{B}_{k}(\mathrm{t})$ is the aggregate bandwidth consumed by already admitted flows of class $k ; \mathrm{B}_{i}$ is the current bandwidth (admission) threshold for class $i$ (where $i \neq k$ ); $\mathrm{B}_{\mathrm{T}}$ is the maximum total bandwidth available to all traffic classes in the LSP; $\mathrm{B}_{i R E S}$ is the 'default' bandwidth reservation in the LSP for class $i$ traffic as set by the $\mathrm{CN}$ operator and $\mathrm{B}_{\mathrm{T}}=\sum_{i=1}^{N} \mathrm{~B}_{i R E S}$.

Thus, the context information will be maintained for each class of traffic with the following mapping:

TABLE 1: CONTEXT INFORMATION MAPPING FOR CNRB POLICIES

\begin{tabular}{|c|c|c|}
\hline $\begin{array}{c}\text { Utilisation } \\
\text { (context) }\end{array}$ & $\begin{array}{c}\text { Corresponding } \\
\text { parameter }\end{array}$ & Admission threshold \\
\hline High & $\geq \alpha^{*} \mathrm{~B}_{i R E S}$ & Default $\left(\mathrm{B}_{i}=\mathrm{B}_{i R E S}\right)$ \\
\hline Low & $<\alpha^{*} \mathrm{~B}_{i R E S}$ & Optimised $\left(\mathrm{B}_{\imath}=\mathrm{B}_{\mathrm{T}}-\sum_{i=1}^{N} \mathrm{~B}_{i}(\mathrm{t})\right)$ \\
\hline
\end{tabular}

Note that the value of $\alpha(0<\alpha<1)$ is chosen by the operator to delimit the boundary between 'high' and 'low' context variables. The default could be set at 0.5 , i.e. the boundary will be at $50 \%$ utilization of default bandwidth reservation.

As the context variables change, so will the admission threshold configuration according the conditions of the CNRB policy rules specified earlier. Within the policy action part, a configuration decision message corresponding to the respective thresholds will be sent to the relevant RCs at the policy enforcement points (edge routers) with configuration settings for the policing mechanisms that ensure compliance with current bandwidth allocation per class in the CNRB policies (see Fig. 2). Such configuration message could be sent using a standard policy provisioning protocol such as the IETF COPS protocol specified for PDP to PEP communication. Bandwidth utilization measure is provided by the network monitoring entity (NM) as shown in Fig. 2. 


\section{Model formulation}

The analytical model of the CNQF CNRB functionality is built using Continuous-Time Markov Chains (CTMC). This model is then solved numerically to yield steady-state solutions from which performance metrics of interest are derived to predict the behavior of the CNRB functionality under various traffic demands. The mathematical model is formulated as follows: Let the state of the CNRB policy-based admission control functionality at a given time instant $t$ be defined by the total number of active sessions $\mathrm{S}(\mathrm{t})=\sum_{i=1}^{N} \mathrm{~S}_{i}(\mathrm{t})$ in the LSP where $N$ is the total number of network service classes defined in the CNRB policies and $\mathrm{S}_{i}$ is the number of active flows in class $i$. For our CNQF use case scenario, the state of the CNRB can therefore be described by an $\mathrm{N}$-dimensional stochastic process given by the vector $\mathrm{V}_{\mathrm{j}}=\left(\mathrm{S}_{1}, \mathrm{~S}_{2}, \mathrm{~S}_{3}\right)$; where $\mathrm{S}_{1}, \mathrm{~S}_{2}, \mathrm{~S}_{3}$ are the number of admitted active sessions of service classes $\mathrm{C}_{1}$ (voice), $\mathrm{C}_{2}$ (video) and $\mathrm{C}_{3}$ (data) respectively. Specifically, $\mathrm{V}_{\mathrm{j}}$ is a 3 dimensional CTMC process assuming independent and exponentially distributed request inter-arrival intervals and session duration respectively. The CTMC captures CNRB policy-based functionality behavior through state transitions governed by the policy rules and provides the tool for performance measures to be computed from the steady state probabilities. Assuming all the possible states are conveniently ordered from $x=0$ to $K-1$. The steady state probability distribution of the model given by $\mathrm{P}=\left\{\mathrm{P}_{\mathrm{x}}(\mathrm{v})\right\}, \mathrm{x}=0 \ldots \mathrm{K}-1$ is the solution of the matrix equation: $\mathrm{P} . \mathrm{Q}=0$, subject to $\sum_{x=0}^{K-1} \mathrm{P}_{\mathrm{x}}(\mathrm{v})$ $=1$. The elements of the Matrix $\mathrm{Q}$ are derived from the transition rates from a state $\mathrm{v}=\left(\mathrm{S}_{1}, \mathrm{~S}_{2}, \mathrm{~S}_{3}\right)$ in the CTMC to a succeeding state $\mathrm{v}_{+}=\left(\mathrm{S}_{1}+, \mathrm{S}_{2}+, \mathrm{S}_{3}+\right)$ on arrival of a service request, or a preceding state $\mathrm{v}^{-}=\left(\mathrm{S}_{1^{-}}, \mathrm{S}_{2^{-}}, \mathrm{S}_{3^{-}}\right)$on completion of a session. Hence the transitions between CNRB states in the model occur as a result of two policy events; the request arrival and the session completion both of which trigger evaluation of conditions and subsequent configuration actions described earlier depending on whether the utilisation context has changed or not.

The CNRB CTMC is built using the stochastic modelling tool MOSEL [18] which is freely available under the GNU public license (GPL) [19]. MOSEL has been used extensively for performance modelling and evaluation of communication systems and networks (e.g. [18], [20]). $\mathrm{P}_{\mathrm{x}}$ yields the values that allow us to compute the performance metrics of the model. The performance metrics computed from our model include:

- Average bandwidth occupancy in CN LSP per class:

Given by: $\mathrm{b}_{i}\left(\sum_{k=0}^{S_{i M A X}} k P x(v)\right)$ for all possible states $v$; where $\mathrm{b}_{i}$ is the bandwidth requested/allocated to each user in class $i$. $\mathrm{S}_{i M A X}$ is the maximum number of admissible requests for class $i$.

- Mean total bandwidth occupancy:

Given by: $\sum_{i=1}^{3} \mathrm{~b}_{i}\left(\sum_{k=0}^{S_{i M A X X}} k P_{x}(v)\right)$ for all possible states $v$;
- Utilisation:

Given by: Mean total bandwidth occupancy/total available $\mathrm{B}_{\mathrm{T}}$

- $\quad$ Service availability per class (\%)

Given by: $100 * \mathrm{~b}_{i}\left(1-\sum_{k=S i M A X} P x(v)\right)$ for all possible states $v$ in the Markov model; $b_{i}$ is the bandwidth requested/allocated to each user in the class $i$.

In order to evaluate the impact of the context-driven CNRB policies (Rules 1 to 3 ) of section IV B, a comparison is made with the reference scenario where only static 'default' configuration is applied. With 'default' configuration, CNRB contextdriven policies are disabled (i.e. only default admission control thresholds $\mathrm{B}_{i R E S}$ are used). For completeness, we also include a comparison with the baseline scenario where the same traffic load is applied but without CNQF functionality.

\section{Numerical results}

Using the stochastic model described in the previous subsection, we ran experiments with the following model configurations taken for all three scenarios:

Service request arrival rates for $\mathrm{C}_{1}$ voice $\left(\lambda_{1}\right), \mathrm{C}_{2}$ video $\left(\lambda_{2}\right)$ and $C_{3}$ data $\left(\lambda_{3}\right)$, (normalized to voice session duration and total bandwidth $\mathrm{B}_{\mathrm{T}}$ ), were varied from 0.1 to 0.9 to capture a wide range of request demand situations. Mean session duration were taken as: voice: 360s; video: 600s; data: 120s. Bandwidth allocations per user were taken as: voice class: 32 kbps; Video class: 384 kbps; BE class: 256kbps. Because request arrivals and session durations are assumed to be independent, they are all modeled with exponentially distributed request inter-arrival times and session durations respectively.

The test scenario assumes 6.144 Gbps for the LPS capacity (provisioned the edge-to-edge LSP) to which the CNRB policy rules are applied. When a user makes a service request to a WARB or FARB, the end-to-end admission control policy per class is evaluated using the rule:

If $\left\{\min \left(B W_{\text {access }}, B W_{\text {core }}, \ldots\right)>B W_{\text {req }}\right\}$ then admit end user request else reject end user request

Thus, $B W_{\text {core }}$ will depend on the current (context-driven) threshold configuration in the CNRB and hence will influence end-to-end admission control and overall and per-class service availability. $B W_{\text {core }}$ per class will be given by $\mathrm{B}_{i}-\mathrm{B}_{i}(\mathrm{t})$, where $\mathrm{B}_{i}$ is either the 'default' or 'optimised' threshold as given in Table 1 and $\mathrm{B}_{i}(\mathrm{t})$ is the aggregate bandwidth consumption by class $i$ traffic. Note that service requests will typically emanate from multiple access networks attached to the CN LSP and with heavy demand, their aggregate could result in bandwidth constraint in the provisioned LSP even when a WARB/FARB reports bandwidth availability within its access domain.

The following bandwidth reservations were assumed for 'default' configuration in the experiments: voice class $\mathrm{B}_{1 \mathrm{RES}}=$ 3.072 Gpbs (i.e. $50 \%$ of total bandwidth); video class $\mathrm{B}_{2 \mathrm{RES}}=$ $1.536 \mathrm{Gbps}$ ( $25 \%$ ), data class $\mathrm{B}_{3 \mathrm{RES}}=1.536 \mathrm{Gbps}$ (i.e. $25 \%$ ).

From the performance metrics defined in the subsection $\mathrm{C}$, the results obtained from the experiments are presented in Figs. 
3 to 7 . Fig. 3 shows the bandwidth occupancy of voice class sessions for the range of request arrival rates considered. It shows that for the same arrival rate, the application of CNRB context-driven policies resulted in better utilisation for voice class traffic compared to when CNQF functionality is absent. As expected, as voice request demand increases, noticeable improvement is observed until the maximum possible $\mathrm{B}_{1 \mathrm{RES}}$ of 3.072 Gpbs. The same performance curve is achieved with 'default' configuration. This can be attributed to the presence of the other two classes of traffic which had higher bandwidth requirements. From Fig. 4 it can be seen that context-aware CNRB policies provide better bandwidth utilisation for video class traffic especially at increased demand (request arrival rate). Also, it is noticeable that default configuration keeps the video bandwidth occupancy around the default maximum reservation $\mathrm{B}_{2 \mathrm{RES}}=1.536 \mathrm{Gbps}$. By contrast, the scenario with the context-aware policies allows higher bandwidth utilisation well above this limit in all request arrival rates.

For data class, Fig. 5 shows that the dynamic context-aware policies allow better bandwidth utilisation than the default configuration in most cases of low demand range and equals its performance limit at high demand rates. Note how the limit of $\mathrm{B}_{3 \mathrm{RES}}=1.536 \mathrm{Gbps}$ is approached at higher demand by the default configuration case, and how the scenario without $\mathrm{CNQF}$ always performed below this. This indicates that because of lack of class-based default admission threshold reservation in the latter scenario, the video and voice class traffic always starved data class traffic, especially due to their longer session durations. The adaptive CNRB policies allow data class bandwidth utilisation to exceed the maximum reserved limit wherever possible because of the context-driven policy rules.

Looking at Fig. 6 (total bandwidth occupancy), it is clear that the utilisation of the bandwidth approaches the maximum as the request demand increases in the case of context-driven adaptive policies in the $\mathrm{CN}$. In this case, the maximum LSP bandwidth capacity of $6.144 \mathrm{Gbps}$ is reached as the demand increased, highlighting the resource utilisation enhancing potential of context-aware policies in the CNRB operation. The results in Figs. 7 and 8 correspond to the trends noticeable in Figs. 3 and 4 for voice and data class traffic respectively.

The results in Fig. 7 depict higher probability of voice service availability in the provisioned CN LSP for both default and context-aware configurations compared to when CNQF is not used. Also, Fig. 8 depicts, in most cases, better or equal probability of service availability for data requests in the case of context-aware policies compared to the default configuration case. Lower probability of data service availability is observed when CNQF is not used. The results obtained from the model demonstrate the efficacy of CNRB context-aware adaptive policies to enhance end-to-end admission control and improve service availability in converged network scenarios. This is especially more noticeable at high traffic demands.

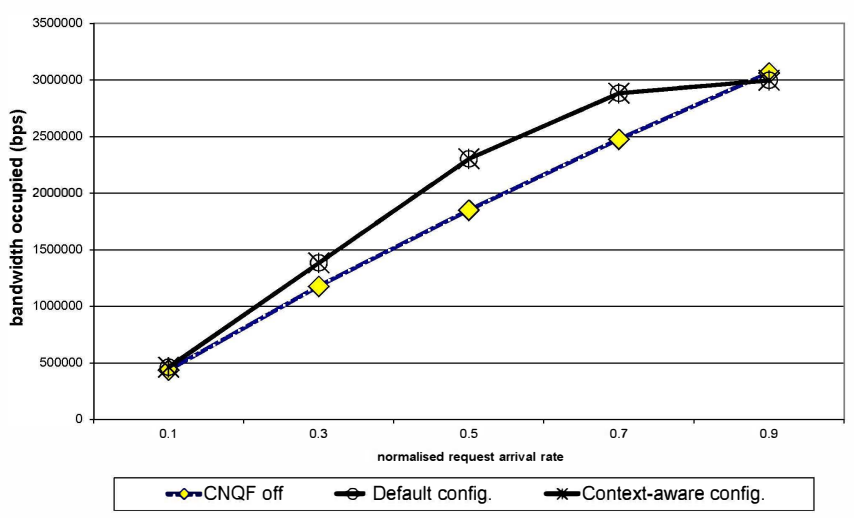

Fig. 3. Voice class bandwidth occupancy

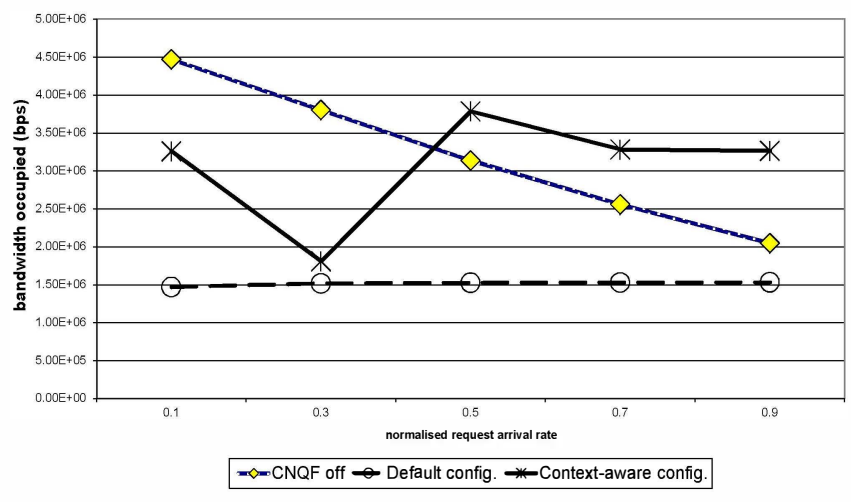

Fig. 4. Video class bandwidth occupancy

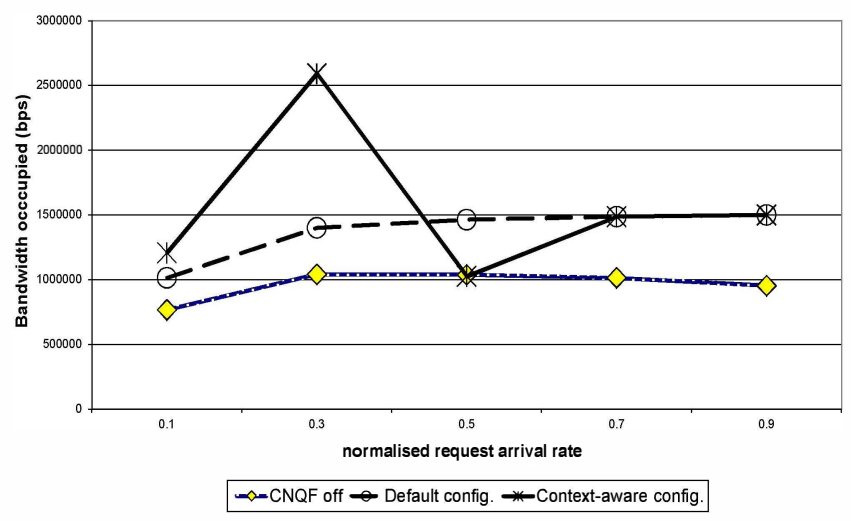

Fig. 5. Data Class bandwidth occupancy

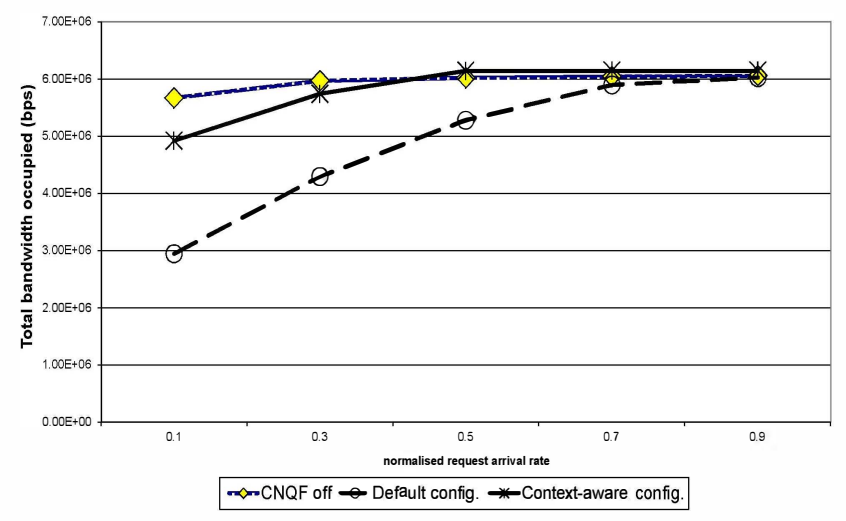

Fig. 6. Total bandwidth occupancy by all three classes 


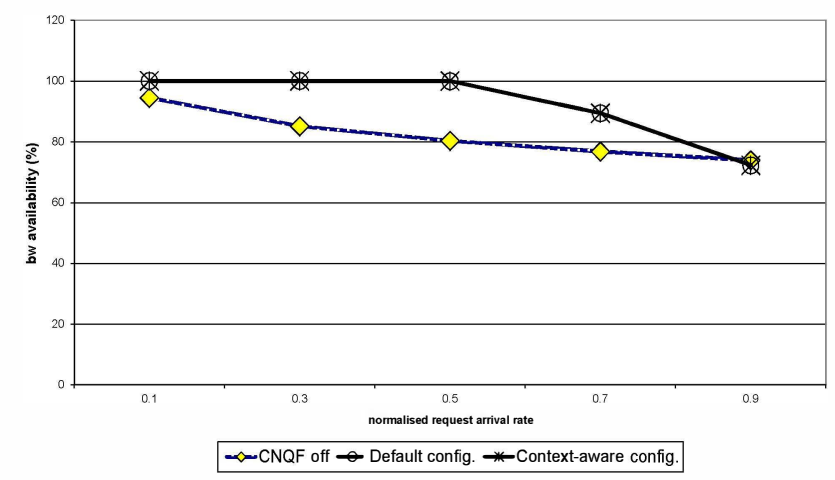

Fig. 7. Voice service availability $(\%)$

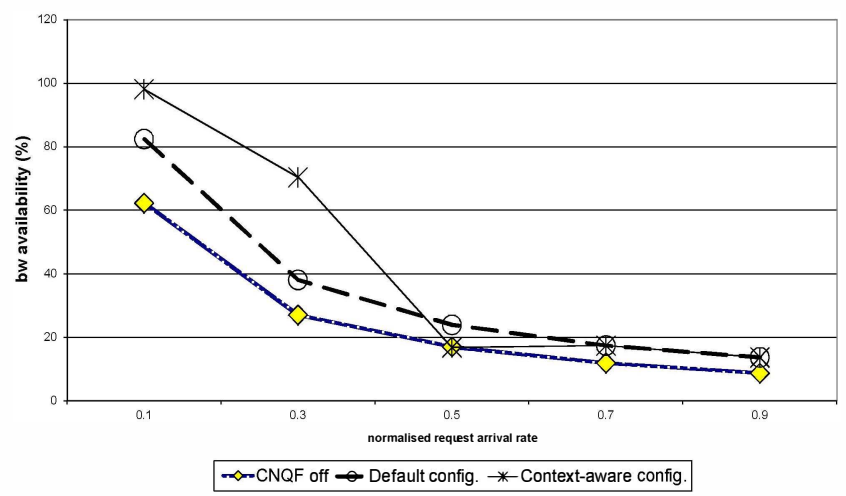

Fig. 8. Data service class availability (\%)

\section{CONCLUSIONS AND FURTHER WORK}

This paper presents an exemplary use case for $\mathrm{CN}$ resource management using $\mathrm{CNQF}$, a policy-based framework for context-aware QoS control in converged networks being developed within our project. CNQF consists of a Resource Management Subsytem, a Context Management and Adaptation Subsystem and a Measurement and Monitoring Subsystem. The functionality of the various policy decision elements within these subsystems could be captured using stochastic-analytic models like the one presented in this paper, providing a costeffective means to evaluate the impact of various policies operating within the system. We have modeled the CNRB using Continuous Time Markov Chains and investigated the impact of a set of context-aware policies for class-based admission control in a hypothetical $\mathrm{CN}$ operator scenario. The results presented demonstrate the efficacy of the CNQF approach. However, the scenario and policies should not be construed as being the only types supported by CNQF. In our future work we aim to investigate for example adaptive QoS control in converged networks driven by user and device-centric context information based on our CNQF policy architecture. Moreover, the model developed in this paper may be used to study other CNQF use case scenarios. The construction of CNQF prototype is currently being undertaken and will be evaluated with real traffic on our experimental converged networks testbeds, the results of which will also be reported in our future work.

\section{ACKNOWLEDGMENT}

This work is funded by the EPSRC-DST India-UK Advanced Technology Centre of Excellence in Next Generation Networks, Systems and Services under grant EP/G051674/1.

\section{REFERENCES}

[1] N. Mistry "The importance of policy-based resource control in future networks". Nortel Technical Journal, issue 4, 2006.

[2] 3GPP TS 23.207: $3^{\text {rd }}$ Generation Partnership Project; End-to-end Quality of Service (QoS) concept and architecture, (Release 9) Dec. 2009.

[3] ETSI ES 282 003: Telecoms and Internet converged Services and Protocols for Advanced Networking (TISPAN); Resource and Admission Control Subsystem (RACS); Functional Architecture, June 2006.

[4] IETF Policy Framework Working Group web page: http://www.ietf.org/wg/concluded/policy.html

[5] S. Y. Yerima, G. P. Parr, C. Peoples, S. McClean, P. J. Morrow, "A Framework for Context-Driven End-to-End QoS Control in Converged Networks" in Proc. $6^{\text {th }}$ International Conference on Network and Service Management, IEEE CNSM 2010, Niagara Falls, Canada.

[6] C.E. Rothenberg and A. Roos. "A Review of Policy-Based Resource and Admission Control Functions in Evolving Access and Next Generation Networks". Journal of Network Systems Management vol. 16 num. 1, pp 14-45, March 2008

[7] J. Strassner. Policy-based network management. Morgan Kaufman Publishers Inc., Los Altos, NJ, ISBN 1-55860-859-1, Sept. 2003.

[8] B. C. Kim et al., "A QoS Framework Design Based on Diffserve and SNMP for Tactical Networks" in. Proc. IEEE MILCOM '08, San Diego, California, Nov. 2008, pp. 1-7.

[9] P. Nanda and A. Simmonds, "A Scalable Architecture Supporting QoS Guarantees Using Traffic Engineering and Policy Based Routing in the Internet" International Journal of Communications, Networks and System Sciences, 2009. pp 583-590.

[10] N. Carrilho and N. Ventura, "Policy-Based Management of a DiffServe Network Using XML Technologies," in Proc. Third International Conference on Web Information Systems and Technologies (WEBIST 2007), Mar. 2007

[11] T. Ahmed, R. Boutaba, A. Mehaoua " A Measurement-Based Approach for Dynamic QoS Adaptation in DiffServe Networks" Computer Communications, Volume 28, Issue 18, pp 2020,- 2033, November 2005.

[12] X. Guo et al. "A Policy-Based Network Management System for IP VPN" International Conference on Communication Technology Proceedings (ICCT 2003), Volume 2, April 2003.

[13] Alex Vallejo, Agustin Zaballos, Jaume Abella, Josep M. Selga, Carles Duz, "Performance of a Policy-Based Management System in IPv6 Networks Using COPS-PR," In Proc. Sixth International Conference on Networking (ICN'07), 2007, pp. 37.

[14] K. El-Khatib, G. Bochmann and A. El Saddik "A QoS-Based Framework for Distributed Content Adaptation" in Proc. $1^{\text {st }}$ IEEE int. Conf. on QoS in Heterogeneous Wired/Wireless Networks (QSHINE'04), Dallas, TX, Oct. 2004, pp. 300-303.

[15] T. Tang, M. Zhengkun, P. Rongqun, "Adaptive service Provisioning Through Context-aware SIP Proxy" in Proc. $4^{\text {th }}$ International Conference on Networking and Services, 2008.

[16] R. Bolla et al., " A Context-aware Architecture for QoS and Transcoding Management of Multimedia Streams in Smart Homes" in Proc. 13th IEEE Intl. Conf. on Emerging Technologies and Factory Automation (ETFA 2008), Hamburg, Germany, Sep.15-18, 2008.

[17] K. Jean, K. Yang, A. Galis, "A Policy Based Context-aware Service for Next Generation Networks" IEE $8^{\text {th }}$ London Communications Symposium, 2003.

[18] K. Al-Begain, et al. "The performance and Reliability Modelling Language MOSEL and its Application," International Journal of Simulation: Systems, Science and Technology vol. 3 No. 3-4, pp. 66-80, 2002.

[19] http://www4.informatik.uni-erlangen.de/Projects/MOSEL/ [Accessed $12^{\text {th }}$ May 2010].

[20] S. Y. Yerima, K. Al-Begain "Novel Radio Link Buffer Management Schemes for End-User Multi-class Traffic in High Speed Packet Access Networks" Wireless Personal Communications Journal, May 29, 2010. 